\title{
A Series of Erector Spinae Plane Blocks Reduced Pain and Opi- oid Consumption in Patients with Post Herpetic Neuralgia: Two Patient Cases
}

\author{
Yuvaraj Kotteeswaran, $M D^{1,2}$, Varuna Manoo, $M D^{1,2}$, Alexander McLaren-Blades, $M D^{1,2}$, Michael \\ Kahn, $M D^{1,2}$, Alexander Huang, $M D^{1,2}$ and Hance Clarke, $M D, P h D^{1,2,3,4^{*}}$
}

${ }^{1}$ Department of Anesthesia and Pain Management, Toronto General Hospital, Canada

${ }^{2}$ Transitional Pain Service, Toronto General Hospital, Canada

${ }^{3}$ Pain Research Unit, Toronto General Hospital, Canada

${ }^{4}$ University of Toronto Centre for the Study of Pain, Canada

*Corresponding author: Hance Clarke, MD, PhD, FRCPC, Staff Anesthesiologist/Director of Pain Services, Medical Director of The Pain Research Unit, Department of Anesthesia and Pain Management, Toronto General Hospital, University Health Network, 200 Elizabeth Street, 3EN-464, Toronto, ON, M5G 2C4, Canada, Tel: +1416-457-4262

\begin{abstract}
Background: Intense, refractory neuropathic pain is an unfortunate complication following a Herpes Zoster $(\mathrm{HZ})$ infection/rash and is regarded as post-herpetic neuralgia (PHN). The risk of PHN increases with age. The erector spinae plane (ESP) block has been demonstrated to block transmission through the thoracic and lumbar spinal nerves. This report describes two patients that were able to significantly reduce their chronic pain medications following a series of ESP blocks.

Methods: ESP blocks were performed in two patients at the transverse process level correlating with their clinical presentation. All the blocks were performed with ultrasound guidance using a previously described technique using 30 $\mathrm{ml}$ of $0.25 \%$ bupivacaine and $80 \mathrm{mg}$ methylprednisolone in a fascial plane deep to erector spinae muscle. Standard monitoring was applied to both patients. A baseline pain score was taken and following the procedure, the patients were given a pain diary to document their pain severity for two weeks. A clinic visit with the completed pain diary was scheduled approximately 2-4 weeks later for block assessment in terms of pain relief, with further clinic follow-up as indicated.
\end{abstract}

Case reports: Both patients had a flare of their PHN following major surgery. The referral to the Transitional Pain Service (TPS) at Toronto General Hospital (TGH) was primarily for help with management of PHN pain and optimization of the patients' pain regimen. Pain medications were being escalated without analgesic benefit and daily activities were becoming negatively impacted. The two patients were scheduled for series of three ESP blocks at intervals of not less than 4 weeks. After every block their pain severity reduced and their medications were able to be tapered gradually.

Conclusions: The results from the above interventions indicate that a series of ESP blocks with local anesthetic and steroid may be considered as a viable therapeutic option in the management of refractory PHN. Concurrent benefits of interventional blocks may include improvements in patients pain scores and functional status, along with the ability for patients to reduce their opioid and other neuropathic pain medication doses and help to reduce the associated side effects.

\section{Keywords}

Post-herpetic neuralgia, Nerve blocks, Opioid analgesics, Interventional pain management, Ultrasound in pain medicine, Erector spinae plane block

\section{Introduction}

Herpes Zoster (HZ), also known as shingles, results from the reactivation of the varicella zoster virus (VZV), which can remain latent in the body for several years after a primary varicella (Chickenpox) infection. Neuro-

Citation: Kotteeswaran Y, Manoo V, McLaren-Blades A, Kahn M, Huang A, et al. (2020) A Series of Erector Spinae Plane Blocks Reduced Pain and Opioid Consumption in Patients with Post Herpetic Neuralgia: Two Patient Cases. Int J Anesthetic Anesthesiol 7:109. doi.org/10.23937/2377-4630/1410109

Accepted: June 24, 2020: Published: June 26, 2020

Copyright: (C) 2020 Kotteeswaran Y, et al. This is an open-access article distributed under the terms of the Creative Commons Attribution License, which permits unrestricted use, distribution, and reproduction in any medium, provided the original author and source are credited. 
pathic pain that persists for months to years after resolution of the $\mathrm{HZ}$ is regarded as Post-herpetic neuralgia (PNH), [1-2]. Approximately 5\%-20\% of those with $\mathrm{HZ}$ go on to develop PHN, [1].

The primary risk factor for the occurrence of $\mathrm{HZ}$ is age. Unfortunately, the incidence of $\mathrm{HZ}$ markedly increases after the age of 50 years correlating with an age-related decline in cell mediated immunity, [2]. The incidence of $\mathrm{HZ}$, and $\mathrm{PHN}$, has also been observed to be increasing. This is partly attributed to an expanding aging population and increasing rates of immunosuppression due to treatment and disease, [3]. Approximately $20 \%$ of people aged $60-65$ years and $30 \%$ of people aged 80 years and older, who have had acute $\mathrm{HZ}$, subsequently develop PHN [4]. Treating the geriatric population with neuropathic pain medication adds another layer of complexity as pharmacokinetics may be altered in this group.

The development of PHN has a detrimental effect on patients' quality of life [5]. Once PHN has been diagnosed, clinicians strive to balance analgesia vs. adverse drug effects. Some accepted treatment modalities for PHN include calcium channel $\alpha 2-\delta$ ligands (gabapentin, pregabalin), tricyclic antidepressants, topical lidocaine, topical capsaicin and opioids [1]. Combination therapies with interventional procedures are also used. Beyond the increase in public health costs, patients suffering with $\mathrm{PHN}$ report poor sleep, reduced ability to work and an overall reduction in their quality of life [6].

The erector spinae plane (ESP) block, first reported in 2016, has been demonstrated to block transmission through the thoracic and lumbar spinal nerves. Additionally, it may also block transmission through sympathetic nerve fibers. In theory therefore, the ESP block should provide pain relief for PHN. A paucity of literature exists describing the successful use of the ESP block in acute $\mathrm{HZ}$ [7] and PHN [8].

In this report, we describe two patients that presented with significant PHN. Following a series of ESP blocks with local anesthetic and steroid their pain improved and they were able to reduce their chronic pain medication.

\section{Case Reports}

\section{Case 1}

An 81-year-old woman with a history of post-herpetic neuralgia 5 years ago underwent a double coronary bypass graft and tricuspid valve repair surgery. She was $157 \mathrm{~cm}$ tall and weighed $70 \mathrm{~kg}$. Preoperatively her functional status was limited and improved significantly following surgery. Following surgery, she unfortunately developed chronic post-sternotomy pain along with a recurrence of her post-herpetic neuralgia symptoms. She was escalated to Oxycodone $60 \mathrm{mg} / \mathrm{day}$ and Pregabalin 75 mg TID without much improvement in pain severity. She was referred to the TPS clinic for management of her PHN and optimization of her analgesic regimen. At presentation, her pain was moderate to severe (NRS 5-7/10) in intensity and described as a constant stabbing, burning and throbbing sensation in the posterior and anterior aspect of the right hemithorax in the distribution of T3-T6. Examination revealed significant allodynia and hyperalgesia along the T4 dermatome. After being reviewed in the TPS clinic, nabilone $0.5 \mathrm{mg}$ BID and a neuropathic topical cream containing ketamine, gabapentin, amitriptyline, baclofen and diclofenac were added to her medication regimen. She showed mild response with no improvement in pain severity. The patient had the course of ESP blocks explained to her and was then scheduled for a series of injections. Informed consent was obtained prior to the performance of each ESP block. Written consent for the publication of these cases was obtained. All the blocks were performed in a designated block bay by the staff anesthesiologist (H.C \& M.K) using the technique as described by Forero $M$, et al. [9]. With the patient seated, scout scan was done using a high frequency ( $\mathrm{L} 12-3 \mathrm{MHz}$ ) linear transducer (Philips CX50, Philips Ultrasound, Andover, MA USA). This was performed to mark the required thoracic spine level by counting spinous process from above. The skin was sterilized with a $2 \%$ chlorhexidine solution and the transducer was placed in a transverse orientation to identify the spinous process, articular process and transverse process. The tip of the desired transverse process was centered on the ultrasound screen, then the probe was orientated to the longitudinal axis and hypoechoic muscle layer of erector spinae muscle was identified overlying the tip of the targeted transverse processes. An $9 \mathrm{~cm} \mathrm{22G}$ block needle (Sonoplex STIM, PAJUNK, Germany) was inserted in-plane in a cephalad to caudal direction to place the needle tip between the fascial layer of erector spinae and the tip of the targeted transverse process. Correct tip position was confirmed by injection of $1 \mathrm{ml}$ of injectate and visualization of hypoechoic fluid spread deep to the erector spinae muscle following which a total of $30 \mathrm{ml}$ of $0.25 \%$ bupivacaine and $80 \mathrm{mg}$ of methylprednisolone was injected. A right ESP block was performed at the T4 level with $30 \mathrm{ml}$ of $0.25 \%$ Bupivacaine and $80 \mathrm{mg}$ methylprednisolone. Thirty minutes later, the patient reported $>50 \%$ pain reduction 1-2/10 for two weeks followed by gradual increase to $5 / 10$. Post-procedure she was given the pain diary to document her pain intensity twice daily. A clinic visit was scheduled approximately 2-4 weeks later in order to assess block efficacy. After 6 weeks, the ESP block was repeated at $\mathrm{T} 4$ with $30 \mathrm{ml}$ of $0.25 \%$ bupivacaine and $80 \mathrm{mg}$ methylprednisolone and she again experienced reduction in pain to $2 / 10$ in intensity for greater than 2 weeks. She returned to the clinic for third ESP blocks after 4 weeks. After every block she showed progress and her medications were tapered gradually. Following her third block her analgesics had been reduced to Oxycodone 30 mg/week, Nabilone 0.25 mg BID, Amitriptyline 
$20 \mathrm{mg}$ QHS and her functional status was significantly improved. At her one-year follow-up visit after her last ESP block, her pain scores remained mild (NRS 2-3/10) and she was engaged with her daily activities.

\section{Case 2}

A 53-year-old man was referred to the TPS clinic two years after a double lung transplant. A pruritic vesicular eruption on the left side of his chest led to a clinical diagnosis of herpes zoster during his postsurgical hospital course. His immunosuppression regimen may have contributed to the development of the $\mathrm{HZ}$ flare and the ultimate development of PHN. A referral to the TPS clinic was made to help manage his PHN. His pain intensity was severe (7-9/10) and he described his pain as an intermittent sharp and stabbing which radiated from the posterior and anterior aspects of his left hemithorax in the T7-T12 dermatomes. He had co-existent allodynia and hyperalgesia in the above dermatomal distributions. Upon presentation to the clinic, he was already on pregabalin (600 mg daily), Tylenol \#3 (acetaminophen/codeine) TID, nabilone $1 \mathrm{mg}$ BID, nortriptyline $10 \mathrm{mg}$ QHS, and a neuropathic topical cream containing ketamine, gabapentin, amitriptyline, baclofen and diclofenac. Given the excessive dose of pregabalin, he experienced significant side effects from the pregabalin including peripheral edema. This gentleman also had the course of ESP blocks explained to him and was then scheduled for a series of injections. Informed consent was obtained prior to each procedure. He also signed the consent form for the publication of his case. Same technique described by Forero $\mathrm{M}$, et al. [9] were used all the times. An ESP block was performed at the level of T8 with $30 \mathrm{ml}$ of $0.25 \%$ bupivacaine and $80 \mathrm{mg}$ methylprednisolone. Thirty minutes later his pain decreased from NRS Score of $9 / 10$ to 2-3/10. Pain diary was handed over to him to document his pain intensity twice daily. A clinic visit was scheduled approximately 2-4 weeks later in order to assess block efficacy. At clinic follow-up at 4 weeks later, he reported the pain of previous intensity (NRS 9/10). A second ESP block was performed at T8 with $30 \mathrm{ml}$ of $0.25 \%$ Bupivacaine and $80 \mathrm{mg}$ Methylprednisolone, following which he experienced $50 \%$ pain reduction for 3 weeks this time. At a third clinic visit 4 weeks after the second block the ESP block was repeated and provided long lasting analgesia of 3/10 in NRS pain intensity. After each block he showed improvement, his medications were gradually tapered. His pain medications were eventually reduced to pregabalin 75 mg BID, nabilone $0.5 \mathrm{mg} \mathrm{BID}$, duloxetine $30 \mathrm{mg}$ QHS and there was no longer the need to use opioid analgesics. He had excellent pain control with this regimen for 8 months, unfortunately he had to undergo a second double lung transplant at that time.

\section{Discussion}

The pathogenesis of PHN is multifactorial. Damage to peripheral and central neurons is a consequence of the inflammatory response that occurs with zoster reactivation and migration along spinal nerves. As a result, nerve fibers (both peripheral and central) may develop lower action potential thresholds leading to spontaneous discharge and disproportionate responses to stimuli (Allodynia and Hyperalgesia).

Risk factors for developing PHN after latent herpes zoster infection include older age, female sex, presence of a prodrome, greater rash severity and greater acute pain severity [10]. The following risk factors explain the fact that the first patient in our case report developed recurrence of PHN along with chronic post-surgical pain. In a cohort study by Forbes, et al. [11], he concluded PHN risk was increased for a number of patient characteristics and comorbidities, notably with age and among those with severe immunosuppression. The second patient described herein developed PNH after the initiation of immunosuppression therapy following his lung transplantation.

PHN management is tailored to the individual patient, but usually involves a multidisciplinary approach that includes optimizing oral analgesics, performing various interventional procedures and psychotherapy. Interventional procedures trialed to date include intercoastal blocks, [12], paravertebral blocks, [13], epidural blocks, [14], and dorsal root ganglion destruction or ablation, [15]. Recently, spinal cord stimulation and peripheral nerve stimulation has demonstrated promise [16]. Despite the lack of a high level of evidence most of these procedures are relatively invasive, costly to implement and technically complex.

ESP blocks under ultrasound guidance are procedures that involve depositing local anesthetics into a paraspinal tissue plane that is distant from the pleura and neuraxial structures. Its safety profile is excellent and local anesthetic spreads readily in the erector spinae plane. All patients will get IV access, baseline vital monitoring including ECG, heart rate, pulse oximetry continuously and non-invasive blood pressure were instituted every 5 minutes in all patients from the beginning of the procedure till block completion. In our setup, the volume of local anesthetics used is based upon the patient weight, maximum dose and toxicity profile of the local anesthetic used. Bupivacaine is generally used in our practice; we dilute the concentration according to required volume to be injected so that the total dose is less than $3 \mathrm{mg} / \mathrm{kg}$. Our block room is equipped with necessary equipment and drugs including Intralipid $20 \%$ to manage the rare complications such as local anesthetic systemic toxicity. All the patients were monitored for complications for a total of $30 \mathrm{~min}$ before being sent to home. A volume of $20-30 \mathrm{~mL}$ in adults produces predictable and extensive cephalo-caudal spread and anesthesia of several dermatomes [17]. A cadaveric study demonstrated that the injectate from ESP blocks involves both the ventral and dorsal ramus of correspond- 
ing spinal nerves, producing loss of sensation over the posterior as well as anterolateral thorax, [18].

The ESP Block has been successfully used as an effective therapeutic option to treat pain from acute $\mathrm{HZ}$ in the emergency department [7]. Beyond a reduction in acute pain, the hope was to prevent $\mathrm{HZ}$ from developing into PHN [19]. There are potentially three mechanisms involved in the pathophysiologal development of PHN. One possibility is that irritated nociceptors induce and maintain sensitization in the spinal dorsal column, [20]. Second, pain could result from a massive degeneration of both myelinated and unmyelinated primary afferents, [21]. Finally, loss of nociceptive primary afferents induces a synaptic reorganization in the dorsal horn, [22]. One or multiple mechanisms can co-exist in a patient [20-23]. In the current report, we elected to perform series of three ESP blocks for a prolonged prevention of peripheral and central sensitization. It is possible that pain free period interrupted the established reverberatory neural circuit between the nociceptors, the central nervous system and the motor unit resulting in pain alleviation [24]. This resulted in attenuation of increased sensitivity to painful stimuli and subsequent central sensitization (Allodynia and Hyperalgesia).

The interventional practice outlined herein with local anesthetic and steroids was able to achieve good efficacy and reasonable long-term effects. Neuraxial steroids are widely used in chronic pain management for its' anti-inflammatory properties. There is the longheld theory that steroids cause suppression of firing from activated and inflamed nerves, and modulation of the afferent pathway. However injecting steroids has its own side effects, [25]. According to the Spine Intervention Society (SIS) recommendations, steroids injection should be repeated with minimum interval of 3 weeks to alleviate the risks of osteoporosis, HPA axis dysregulation and glycemic control issues [26]. Both patient treatment courses adhered to these guidelines.

The insertion of indwelling catheters has been suggested as a modality to prolong ESP block effects, however this is not possible or practical in ambulatory chronic pain setting. Even though similar analgesic effect can be achieved from epidural or paravertebral blocks, the ESP blocks offers the advantage of being a simple technique, very easily performed, with a predictable and extensive spread of local anesthetics to include multiple dermatomes. The erector spinae muscle is a paraspinal muscle that extends along the entire thoracic vertebral column on either side of the spine. The erector spinae muscle along its entire length is enclosed in a thoracolumbar fascia which acts as a conduit for spread of local anesthetics along the multiple thoracic dermatomal levels [27]. While conducting the ESP blocks, there is a clear end point of the transverse process for needle insertion, so the risk of dural puncture or pneumothorax is much lower than an epidural or paravertebral procedure.

\section{Conclusion}

The results observed with the two patients presented indicate that a series of ESP blocks with local anesthetic and steroid may be a valuable therapeutic option in the management of PHN. Concurrent benefits of interventional blocks may include improvements in patients' pain scores and functional status, along with the ability for patients to reduce their pain medication doses and reduce side effects. This highlights the potential for erector spinae plane blocks as a therapeutic modality in the armamentarium for the management of PHN. The clinical utility of the ESP block for patients suffering with $\mathrm{PNH}$ needs to be further investigated in future studies.

\section{Acknowledgements}

None.

\section{Approval from Authors}

All authors have read and approved the paper.

\section{Declarations of Interest}

None.

\section{Conflict of Interest}

Authors declare no conflicts of interest.

\section{Informed Consent}

Written informed consent has been obtained from both participants included in this case series.

\section{Ethical Approval}

Institutional research ethics board approval is not required for case reports and thus was not obtained.

\section{Authors Contribution}

Yuvaraj Kotteeswaran helped to acquisition of data, review the literature and write the manuscript.

Varuna Manoo helped to write the manuscript.

Alexander McLaren-Blades helped to review the literature and write the manuscript.

Michael Kahn helped to write the manuscript.

Alexander Huang helped to write the manuscript.

Hance Clarke helped to design the case report, review the literature and write the manuscript.

\section{References}

1. Mallick-Searle T, Snodgrass B, Brant J (2016) Postherpetic neuralgia: Epidemiology, pathophysiology, and pain management pharmacology. J Multidiscip Healthc 9: 447-454.

2. Kawai K, Gebremeskel BG, Acosta CJ (2014) Systematic review of incidence and complications of herpes zoster: Towards a global perspective. BMJ Open 10: e004833.

3. Marra F, Chong M, Najafzadeh M (2016) Increasing incidence associated with herpes zoster infection in British Columbia, Canada. BMC Infect Dis 16: 589. 
4. Fashner J, Bell AL (2011) Herpes zoster and postherpetic neuralgia: Prevention and management. Am Fam Physician 83: 1432-1437.

5. Makharita MY (2017) Prevention of post-herpetic neuralgia from dream to reality: A ten-step model. Pain Physician 20: E209-E220.

6. Yu S-Y, Fan B-F, Yang F, DiBonaventura M, Chen Y-X, et al. (2019) Patient and economic burdens of postherpetic neuralgia in China. Clin Outcomes Res CEOR 11: 539-550.

7. Tekin E, Ahiskalioglu A, Aydin ME, Sengun E, Bayramoglu A, et al. (2019) High-thoracic ultrasound-guided erector spinae plane block for acute herpes zoster pain management in emergency department. Am J Emerg Med 37: 375. e1-375.e3.

8. Ueshima $\mathrm{H}$, Otake $\mathrm{H}$ (2018) Erector spinae plane block for pain management of wide post-herpetic neuralgia. J Clin Anesth 51: 37 .

9. Forero M, Rajarathinam M, Adhikary S, Chin KJ (2017) Erector spinae plane (ESP) block in the management of post thoracotomy pain syndrome: A case series. Scand J Pain 17: 325-329.

10. Jung BF, Johnson RW, Griffin DRJ, Dworkin RH (2004) Risk factors for postherpetic neuralgia in patients with herpes zoster. Neurology 62: 1545-1551.

11. Forbes HJ, Bhaskaran K, Thomas SL, Smeeth L, et al (2016) Quantification of risk factors for postherpetic neuralgia in herpes zoster patients: A cohort study. Neurology 87: 94-102.

12. Doi K, Nikai T, Sakura S, Saito $Y$ (2002) Intercostal nerve block with $5 \%$ tetracaine for chronic pain syndromes. J Clin Anesth 14: 39-41.

13. Ji G, Niu J, Shi Y, Hou L, Lu Y, Xiong L (2009) The effectiveness of repetitive paravertebral injections with local anesthetics and steroids for the prevention of postherpetic neuralgia in patients with acute herpes zoster. Anesth Analg 109: 1651-1655.

14. Dureja GP, Usmani H, Khan M, Tahseen M, Jamal A (2010) Efficacy of intrathecal midazolam with or without epidural methylprednisolone for management of post-herpetic neuralgia involving lumbosacral dermatomes. Pain Physician 13: $213-221$.

15. Chun-jing H, Yi-ran L, Hao-xiong N (2012) Effects of dor- sal root ganglion destruction by adriamycin in patients with postherpetic neuralgia. Acta Cir Bras 27: 404-409.

16. Lin C-S, Lin Y-C, Lao H-C, Chen C-C (2019) Interventional treatments for postherpetic neuralgia: A systematic review. Pain Physician 22: 209-228.

17. Chin KJ, Adhikary S, Sarwani N, Forero M (2017) The analgesic efficacy of pre-operative bilateral erector spinae plane (ESP) blocks in patients having ventral hernia repair. Anaesthesia 72: 452-460.

18. Forero M, Adhikary SD, Lopez H, Tsui C, Chin KJ (2016) The erector spinae plane block: A novel analgesic technique in thoracic neuropathic pain. Reg Anesth Pain Med 41: 621-627.

19. Park YJ, Kang SJ, Kim YH, Lee JH (2019) Successful application of the erector spinae plane block for the management of zoster-associated pain. J Clin Anesth 60: 70-71.

20. Fields HL, Rowbotham M, Baron R (1998) Postherpetic neuralgia: Irritable nociceptors and deafferentation. Neurobiol Dis 5: 209-227.

21. Haanpää M, Laippala $P$, Nurmikko T (2000) Allodynia and pinprick hypesthesia in acute herpes zoster, and the development of postherpetic neuralgia. J Pain Symptom Manage 20: $50-58$.

22. Baron R, Saguer M (1993) Postherpetic neuralgia. Are C-nociceptors involved in signalling and maintenance of tactile allodynia? Brain J Neurol 116: 1477-1496.

23. Kelly DJ, Ahmad M, Brull SJ (2001) Preemptive analgesia I: Physiological pathways and pharmacological modalities. Can J Anaesth J Can Anesth 48: 1000-1010.

24. Naja ZM, Maaliki H, Al-Tannir MA, El-Rajab M, et al. (2006) Repetitive paravertebral nerve block using a catheter technique for pain relief in post-herpetic neuralgia. $\mathrm{Br} \mathrm{J}$ Anaesth 96: 381-383.

25. Cohen SP, Bicket MC, Jamison D, Wilkinson I, Rathmell JP (2013) Epidural steroids: A comprehensive, evidence-based review. Reg Anesth Pain Med 38: 175-200.

26. Schneider BJ, Mattie R, Smith C (2019) Spine Intervention Society's Patient Safety Committee. Cumulative Lifetime Steroid Exposure via Epidural Administration. Pain Med Malden Mass.

27. Willard FH, Vleeming A, Schuenke MD, Danneels L, Schleip $R$ (2012) The thoracolumbar fascia: Anatomy, function and clinical considerations. J Anat 221: 507-536. 